A Revolute Joint With Linear Load-Displacement Response for Precision Deployable Structures

\author{
Mark S. Lake \\ NASA Langley Research Center \\ Hampton, VA \\ And \\ Peter A. Warren and Lee D. Peterson \\ University of Colorado \\ Boulder, $\mathrm{CO}$
}

Presented at the 37th AIAA/ASME/ASCE/AHS/ASC Structures, Structural Dynamics, and Materials Conference

AIAA Paper No. 96-1500

Salt Lake City, Utah

April 15-17, 1996 



\title{
A Revolute Joint With Linear Load-Displacement Response for Precision Deployable Structures
}

\author{
Mark S. Lake* \\ NASA Langley Research Center, Hampton, Virginia, 23681 \\ Peter A. Warren ${ }^{\dagger}$, and Lee D. Peterson ${ }^{\ddagger}$ \\ University of Colorado, Boulder, Colorado, 80309
}

\begin{abstract}
NASA Langley Research Center is developing key structures and mechanisms technologies for micron-accuracy, in-space deployment of future science instruments. Achieving micron-accuracy deployment requires significant advancements in deployment mechanism design such as the revolute joint presented herein. The joint presented herein exhibits a load-cycling response that is essentially linear with less than two percent hysteresis, and the joint rotates with less than one in.oz. of resistance. A prototype reflector metering truss incorporating the joint exhibits only a few microns of kinematic error under repeated deployment and impulse loading. No other mechanically deployable structure found in the literature has been demonstrated to be this kinematically accurate.
\end{abstract}

\section{Introduction}

A continuing challenge for scientists and technologists is to reduce significantly the cost of spacecraft and science instruments while increasing their performance through the use of advanced technologies. technology can enable reflector apertures to exceed payload shroud diameters. Thus, a given reflector aperture can be packaged within a smaller and less expensive launch vehicle, or conversely, a given launch vehicle can accommodate an instrument with a larger 
whereas reflectors for science instruments operating in the teraHertz range require surface accuracies of microns (or less). Nonlinearities in present state-of-the-practice deployment mechanisms introduce dimensional uncertainties that limit the accuracy of deployable structures to approximately 100 microns (Ref. 2). Improving deployment accuracy by two orders of magnitude requires significant advancements to be made in the design of precision deployment mechanisms and the understanding of sub-micron nonlinear response of these mechanisms.

The revolute (i.e., hinged) joint described herein $\$$ has been developed at the NASA Langley Research Center for application in many precision deployable structures including metering trusses for segmented telescope reflectors. The objectives of this paper are to: 1) discuss the nonlinear structural response of conventional revolute joint designs and explain how these nonlinearities affect deployment accuracy; 2) describe the features designed to eliminate nonlinearities in the revolute joint described herein; and 3) present test results for individual joints and a prototype metering truss incorporating multiple joints to show the feasibility of the new design.

\section{Background Behavior of Conventional Revolute Joints}

Revolute joints are necessary in all deployable structures to allow folding and unfolding of components. Conventional revolute joints consist of a tang which rotates around a clearance-fit pin and clevis assembly. As shown in Fig. 2, the pin-clevis assembly surrounds the tang resulting in a geometry that is symmetric about two perpendicular planes passing through the center of the joint. One symmetry plane is perpendicular to the pin axis, and the other symmetry plane contains the pin axis. This symmetry insures

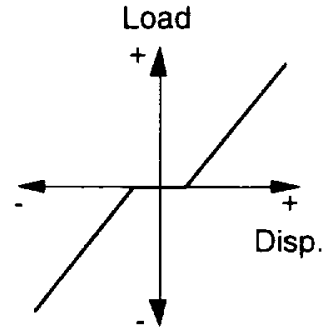

(a) Freeplay

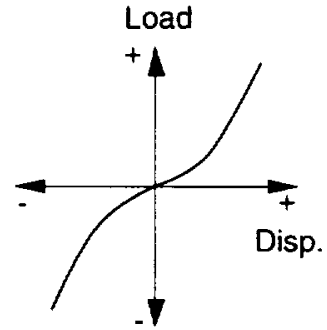

(b) Changing stiffness

that the joint will not bend laterally as axial tension and compression loads are applied; and the symmetry places the pin in a state of double shear giving the joint higher stiffness and strength than a joint in single shear.

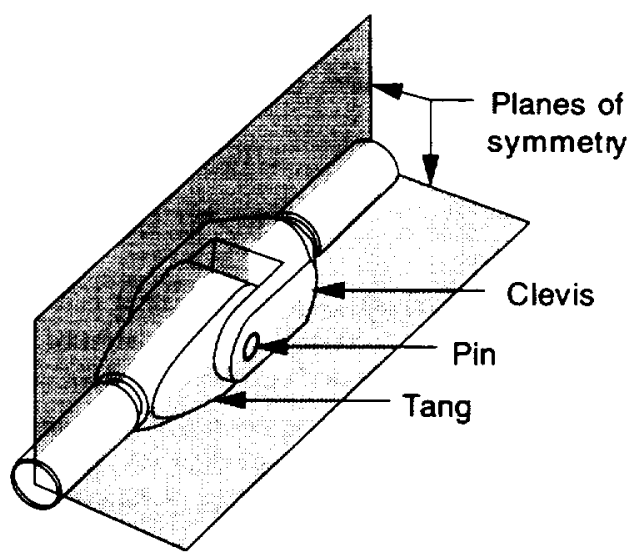

Fig. 2 Conventional pin-clevis joint.

Under tension-compression load cycling, conventional pin-clevis joints exhibit four types of nonlinear load-displacement response as shown in Fig. 3: a) freeplay due to clearances between the pin, tang, and clevis; b) changes in stiffness due to the nonlinear contact between the pin, the tang, and the clevis; c) unequal tension and compression stiffnesses (referred to herein as bilinearity) due to different tension and compression load paths through the clevis and the tang; and d) hysteresis due to friction between the joint components. Numerous studies (e.g., Refs. 3 through 6) have shown it to be essentially impossible to predict nonlinear joint response analytically. Current mathematical models of nonlinear joint phenomena involve numerous empirical parameters whose values are highly dependent on specific test conditions.

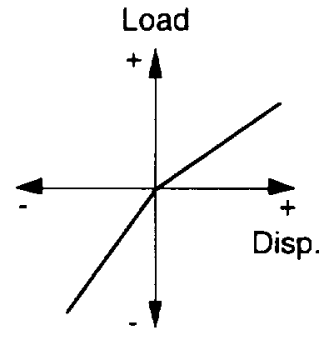

(c) Bilinearity

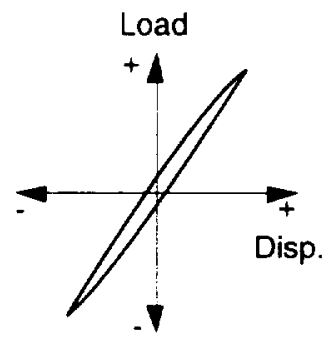

(d) Hysteresis

Fig. 3 Nonlinear load-displacement response of conventional pin-clevis joints.

\footnotetext{
${ }^{\S}$ Invention Disclosure Case No. LAR 15300-1 has been filed with the NASA Langley Patent Counsel.
} 


\section{Effect of Joint Nonlinearities on the Dimensional Accuracy of Deployable Structures}

In addition to introducing uncertainty in the response of a deployable structure, joint nonlinearities can be a source of dimensional errors in a deployable structure. For example, the dimensional accuracy of a deployable structure with freeplay in its joints is limited by the magnitude of the freeplay. Data presented in a subsequent section indicate that friction-induced hysteresis within joints can also produce dimensional errors in a deployable structure.

Dimensional errors caused by joint freeplay and hysteresis are defined herein as "kinematic errors." Studies have shown that freeplay-induced kinematic errors can only be compensated for by using active devices which "artificially" stiffen the joints and essentially eliminate the freeplay (Ref. 7). At present,

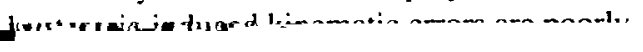

to consider joint and mechanism concepts that can be preloaded locally to minimize nonlinearities and kinematic errors, rather than system concepts that are preloaded globally.

Kinematic errors represent a subset of all errors which define a structure's dimensional accuracy. Other error types are strain-induced errors (mechanical. thermal, and hygroscopic) and fabrication errors. Even if kinematic errors are reduced to a small fraction of the dimensional accuracy requirement, fabrication and straininduced errors must be controlled to achieve the desired dimensional accuracy. The following two general principles can be applied to minimize the cost and risk of passively and actively compensating for fabrication and strain-induced errors (Ref. 9):

- The cost and risk of active compensation of strain-induced errors is minimized if the

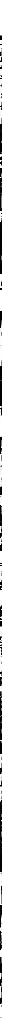



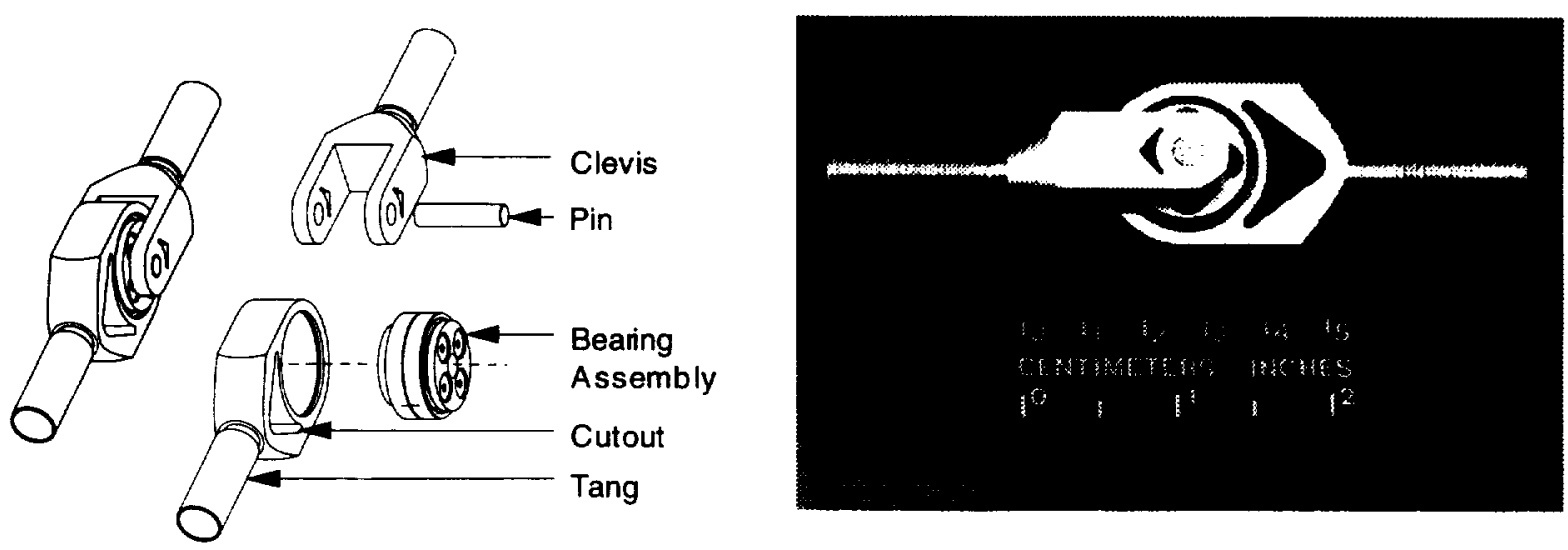

Fig. 4 Linear revolute joint.

The bearing assembly, shown in Fig. 5, consists of a hub and preload plate which retain and preload a commercially manufactured pair of precision angularcontact bearings. The hub is machined with an outer diameter that makes a slip-fit with the inner race of the angular-contact bearing. A raised lip is machined on the outer surface of the hub to retain the inner race of one bearing and react the preloading force applied by the preload plate, through the other bearing. The preload plate is attached to the hub using four small machine screws. As these screws are tightened, the outer lip of the preload plate makes contact with the inner race of one of the angular-contact bearings. The clamping force generated by the four machine screws is applied solely to the inner races of the angular-contact bearings, thus preloading the bearings according to the bearing manufacturer's specifications. Prior to preloading the bearings onto the hub, a thin-film liquid adhesive is applied between the inner bearing races and the hub to insure intimate contact and to eliminate any potential for freeplay: Once completed, the bearing assembly is bonded into the cylindrical cavity of the tang with the same thin-film liquid adhesive (see Fig. 4).

The tang incorporates a threaded shaft for attachment to other structural components. A cutout is machined into the body of the tang to divide the joint axial load into two paths. The load path is divided in this manor to ensure that the tension stiffness and the compression stiffness of the tang are equal. Details of the cutout

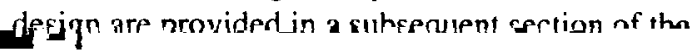

hub. The clevis arms also have cutouts that are designed to divide the axial load through the joint ensuring equal stiffnesses in tension and compression and eliminating bilinearity.

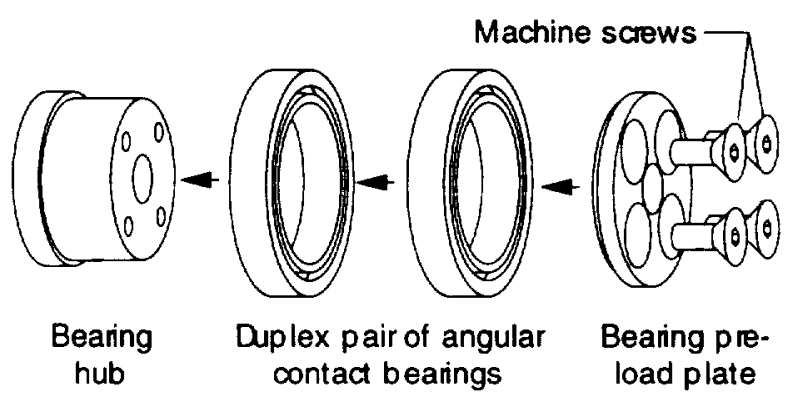

Fig. 5 Bearing assembly.

\section{Preloaded Bearings}

The source of most nonlinearities in conventional pin-clevis revolute joints is the pin and the pin-clevis interface (Ref. 3). As mentioned previously, traditional pin-clevis joints require clearance between the pin and clevis (i.e., the pin diameter is less than the clevis-hole diameter) to allow free rotation of the joint. Unfortunately, this clearance introduces both freeplay and variable stiffness, associated with nonlinear pin-hole contact stresses, into the load-displacement response of the joint (see Fig. 3). To minimize these nonlinear effects, the preloaded pair of angular-contact bearings present paper.

The clevis is a split-fork design which surrounds and attaches to the bearing assembly and includes a threaded shaft for attachment to other structural components. The holes through the clevis arms are sized for a pressfit with the pin, and final assembly of the joint is achieved by simultaneously pressing the pin through these clevis arms and the central hole of the bearing of a simple pin.

Angular-contact bearings are often referred to as "duplex" bearings because they are manufactured in matched pairs and installed back-to-back so that a known preload is developed between the races and the balls as the inner races of the bearings are clamped together. Because of this preloading process, all clearance (freeplay) is eliminated between the balls and 
the races. However, the Hertzian-contact stress between the balls and the races introduce the possibility of nonlinearity in the load-displacement response of the bearing (Ref. 10). To minimize this effect, the bearing set used in the linear revolute joint has a relatively large diameter (approximately 0.75 in.) which maximizes both the size of the balls and the number of balls which carry load. Finally, the bearings requires only about 0.5 in.-oz. of applied torque to overcome the operating friction, thus minimal force would be required to deploy a structure using these joints (Ref. 11).

Although preloaded angular-contact bearings are commonly used in high-precision articulating mechanisms (Ref. 12), no examples of the use of angular-contact bearings in the design of deployable structures have been found in the literature. The present application of angular-contact bearings is considered novel in the sense that the bearings are used not only to achieve high precision, but also to minimize loaddisplacement response nonlinearities.

\section{Stiffness Tailoring of the Tang and Clevis}

In a typical pin-clevis revolute joint, bilinearity is caused by differences between the tension and compression load paths resulting from pin-clevis and pin-tang interfaces. In general, these joints have a more direct load path in compression than in tension, and, as a result, exhibit a higher compression stiffness than tension stiffness.

The cutout shown in Fig. 6 divides the load paths through the tang to insure equal tension and compression stiffnesses (similar features apply to the clevis arms). The cutouts effectively reduce the compression stiffness of the joint by forcing the compression load path to divide rather than pass in a straight line to the bearing. To eliminate bilinearity. the cutouts are sized such that the compression stiffness of the tang equals the tension stiftiness of the tang. assuming the bearing-tang interface carries no radial tension. This assumption practically insures equal tension and compression stiffnesses regardless of the radial stiffness of the bearing-tang interface adhesive.

A limited parametric study was conducted using a two-dimensional finite element model of a generic tang in which tension and compression loads were applied to the free end of the tang (left end in Fig. 6) and reacted by radial compressive pressure on the inner boundary of the bearing cavity. These finite element analyses demonstrate that nearly equal tension and compression stiffnesses result if the cutout is sized and shaped as shown in Fig. 6. The cutout should extend approximately 45 degrees in each direction from the centerline of the joint, and it should he at least as deep along the joint axis as the inner radius of the bearing cavity. Also, the region between the bearing cavity and the cutout should be thinned to approximately $70 \%$ of the nominal thickness outside of the cutout. Other cutout shapes that might be tailored to eliminate bilinearity were not considered in the present study.

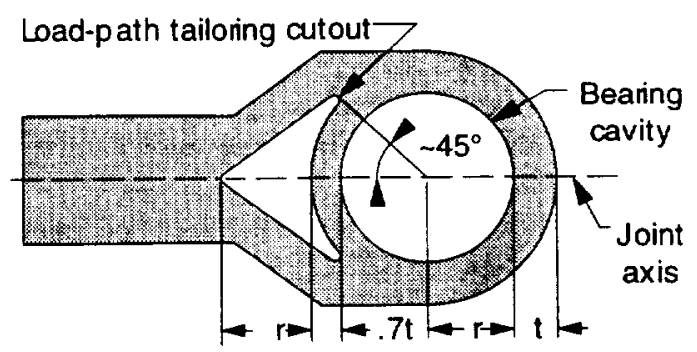

Fig. 6 Tang cutout for equal tension and compression stiffness.

\section{Test Results}

\section{Component-Level Joint Tests}

A few prototype joints (e.g., see Fig. 4) were fabricated for quasi-static axial load-cycle testing using the setup shown in Fig. 7. Each joint was threaded into adapter fittings and installed in a 50,000-lb.-capacity tension/compression test machine. A high-sensitivity 500-lb.-capacity load cell was installed between the joint and the test-machine crosshead to measure the total axial load applied to the joint. Center-line axial displacement within the joint was determined by averaging displacement measurements from three highsensitivity linear voltage displacement transducers positioned equidistantly around the joint. During each test the joint was quasi-statically cycled between $50 \mathrm{lb}$. of tension and compression loads and between $100 \mathrm{lb}$. of tension and compression loads. Joints having two different hearing-preload values were tested.

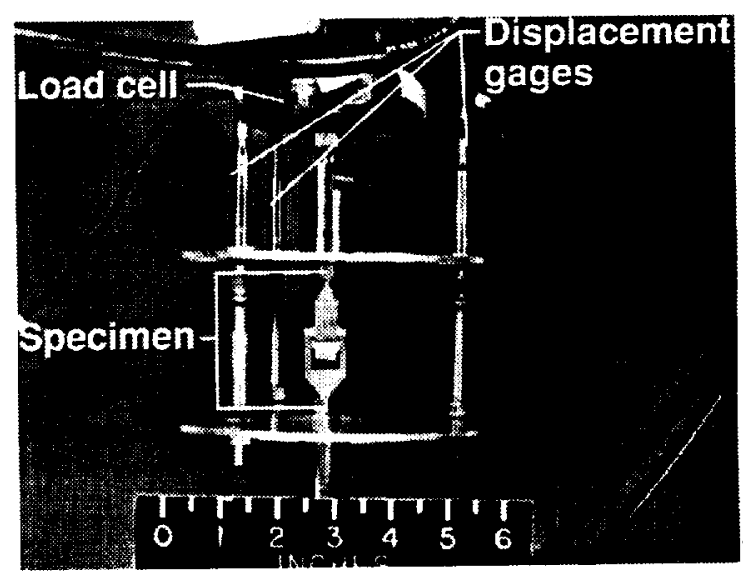

Fig. 7 Axial load-cycle test setup. 
Representative load-displacement responses are plotted in Figs. 8 and 9 for joints with bearing preloads of 10 to $15 \mathrm{lb}$. and 25 to $32 \mathrm{lb}$., respectively (the range in the preload is a bearing manufacturer's specification). Also shown on the right side in these figures are the load-displacement responses after best-fit straight lines have been subtracted from the total displacement. The remaining displacement is the joint hysteresis. The displacement gages used are only accurate to about 1 micron, hence the noise seen in the hysteresis plots of Figs. 8 and 9 is likely to be from the instrumentation rather than from the response of the joint.

The results shown in Fig. 8, for the joint specimen with a 10 to $15 \mathrm{lb}$. bearing preload, include load cycles of \pm 50 lbs. and \pm 100 lbs. For each load range, the joint was cycled three times, and the results from all load cycles are presented to demonstrate the repeatability of the joint behavior. The load-displacement response is essentially linear with less than $2 \%$ hysteresis. The hysteresis loop is approximately one micron wide for the $\pm 100 \mathrm{lb}$. load cycle, and somewhat narrower for the $\pm 50 \mathrm{lb}$. load cycle. The shape of these hysteresis loops and their scaling with load-cycle magnitude are consistent with the occurrence of localized Coulombic micro-slippage between the balls and races due to the tangential load carried by the ball-race interface under load cycling (Ref. 13). Similar load-cycle tests conducted with a prismatic aluminum rod yielded approximately an order of magnitude less materialinduced hysteresis. These results reinforce the assumption that the hysteresis in the load-displacement response of the joint is due to friction between the balls and races within the bearings.

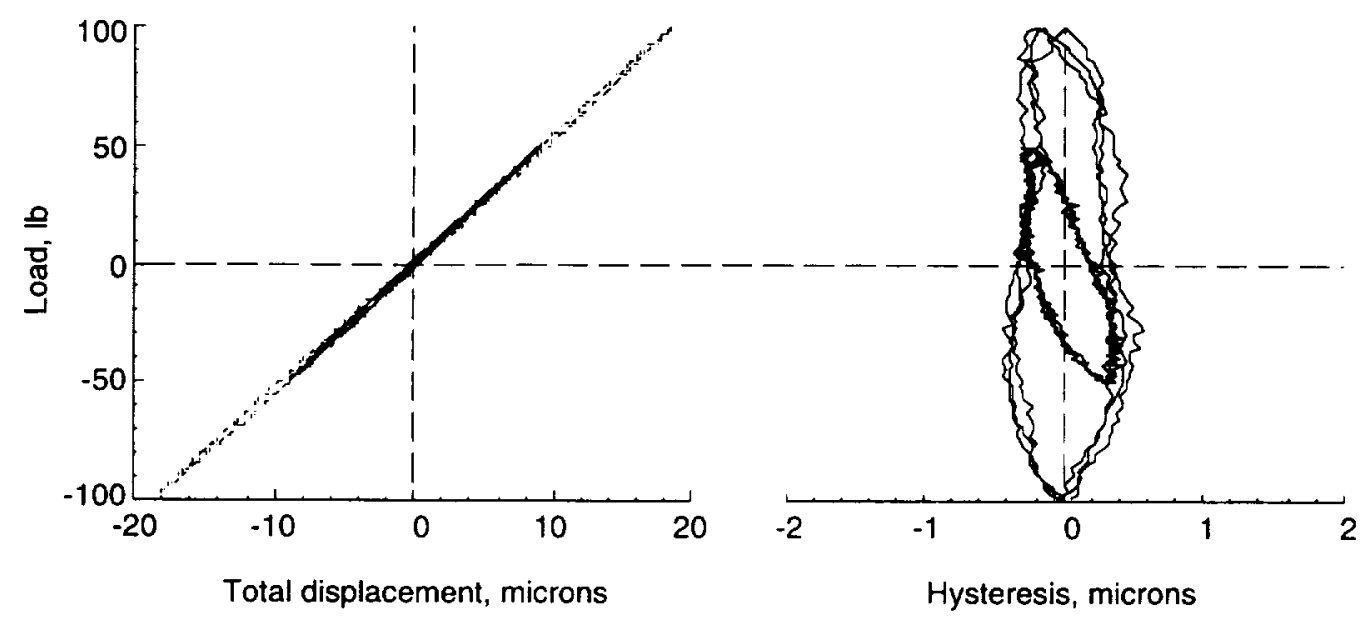

Fig. 8 Load-displacement response and hysteresis loops for a joint with 10 to $15 \mathrm{lb}$. bearing preload.

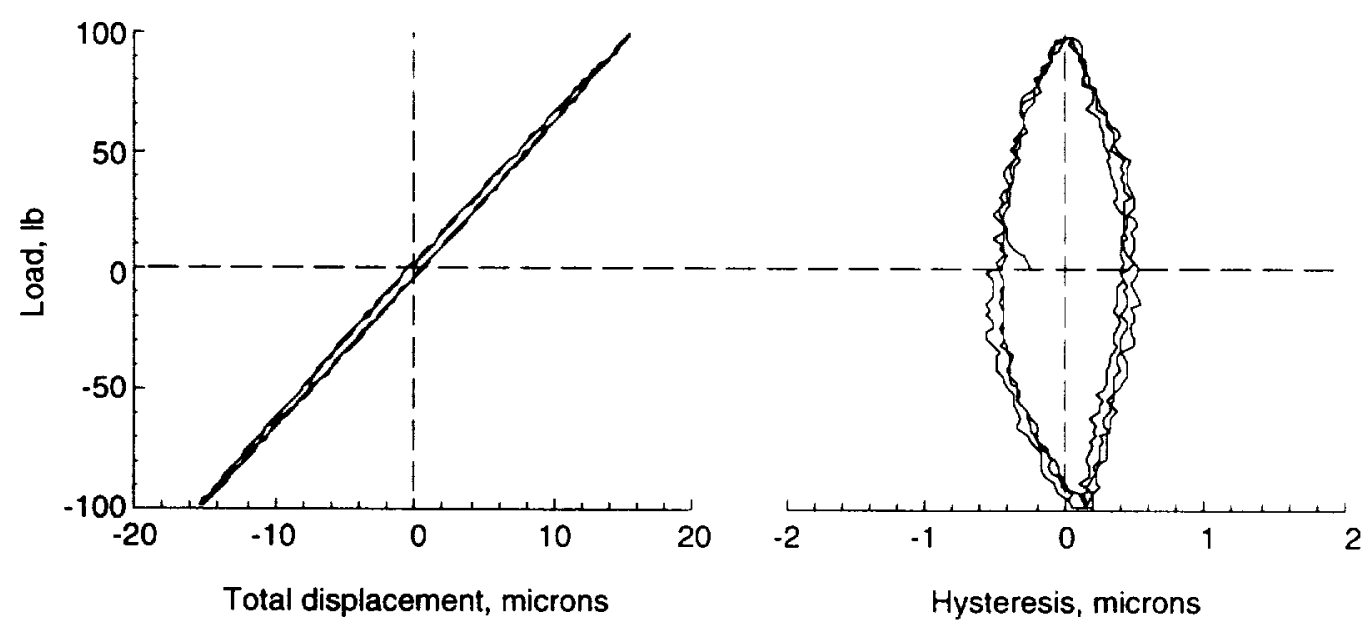

Fig. 9 Load-displacement response and hysteresis loop for a joint with 25 to 32 lb. bearing preload. 
The results for the test joint with a 25 to $32 \mathrm{lb}$. bearing preload shown in Fig. 9 also include three load cycles. Comparing the data presented in Figs. 8 and 9 shows that both the stiffness and the amount of hysteresis in the joint increase slightly with increasing bearing preload. This result is consistent with the nonlinear Hertzian contact condition that exists between the balls and the races; i.e., as the normal force increases at this interface, the stiffness and the tangential friction forces increase as well.

It was noted previously herein that the bearing-clevis and bearing-tang interfaces of the joint can carry tensile load due to the use of a thin-film adhesive, and that this interface effect could cause the joints to exhibit bilinearity due to differences in tension and compression stiffnesses. However, the linearity of the results presented in Figs. 8 and 9 suggests that the cutouts in the tang and clevis arms are effective in eliminating bilinearity in the linear revolute joints without regard to the tensile stiffness at the bearing-tang interface.

\section{Telescope Reflector Metering Truss Tests}

To evaluate the linear revolute joint as part of a structural assembly, a portion of a deployable metering truss for a segmented reflector was fabricated. A photograph of the test article is shown in Fig. 10. This test article represents a portion of the metering truss that supports one reflector panel (one of the six perimeter panels shown in Fig. 1). The metering truss test article incorporates four of the revolute joints shown in Fig. 4. Two of these joints are at the base of the truss and two are at the tip to allow the truss to fold vertically into a narrow package. The test article also incorporates a latch joint that locks the truss in position at the end of deployment. To date, no component-level testing has been performed on the latch joint.

A significant amount of testing has been performed to characterize the structural response and kinematic accuracy of the metering truss test article. In these tests. the positions of key points on the structure were monitored during dynamic loading using an innovative

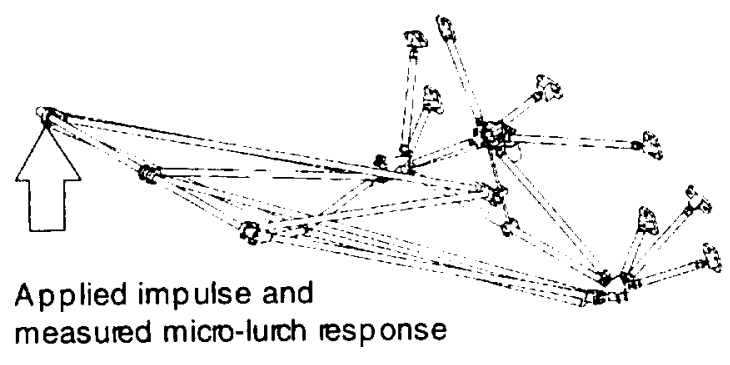

new videographic metrology system with approximately ten-nanometer resolution (Ref. 14). Results from these tests indicate that the structural response of the test article is linear to within a few microns for both static and dynamic loading, and the test article exhibits only a few microns of kinematic error between successive deployments and impulse loading.

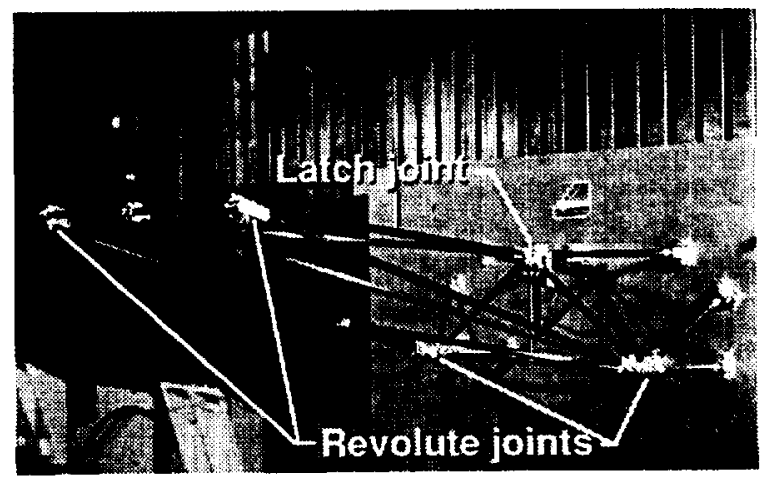

Fig. 10 Precision reflector metering truss fabricated with linear revolute joints.

From these tests, a new nonlinear response phenomenon referred to herein as "micro-lurching" has been discovered which is a change in the equilibrium position of points on the structure following a transient disturbance. In each series of tests, the structure was deployed and then subjected to an impulsive load by a light tap on one of the outboard nodes as shown in the sketch in Fig. 11. The vertical location of the node was determined before applying the impulsive load and after the impulse response attenuated. A typical set of results from these tests (plot in Fig. 11) shows the changes in node location (i.e., micro-lurches) that occur after successive impulses are applied to the test article. The first five impulses after deployment cause a total of about seven microns of micro-lurching in one direction, and the remaining impulses cause random micro-lurches of no more than a few microns each.

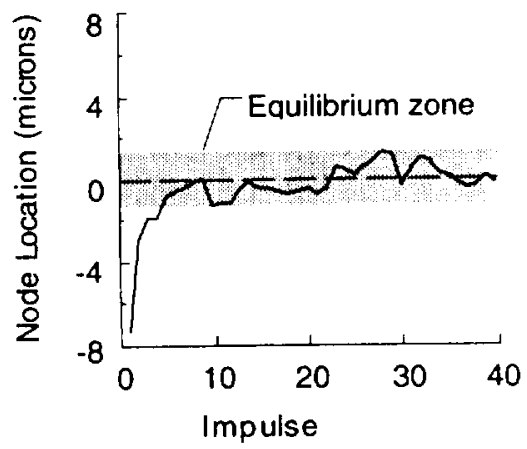

Fig. 11 Typical micro-lurching response of metering truss. 
Preliminary analyses using the simple two-degreeof-freedom dynamic model sketched in Fig. 12 suggest that micro-lurching is a typical nonlinear response in structures that transmit load through Coulombic friction that is schematically parallel to the elastic deformation. Since both the revolute joints and the latch joint transmit load in this manor, it seems plausible that these mechanisms are responsible for micro-lurching. However, additional analyses and tests are necessary to fully characterize the relationship between microlurching and friction-induced hysteresis in the revolute joints or nonlinearities (as of yet uncharacterized) in the latch joint. Furthermore, additional analyses and tests are necessary to determine if micro-lurching is affected by gravity-induced preloading in the structure. These investigations are very important because the stochastic nature of micro-lurching might ultimately prove it to be the response parameter that defines the kinematic accuracy of a precision mechanically deployable structure.

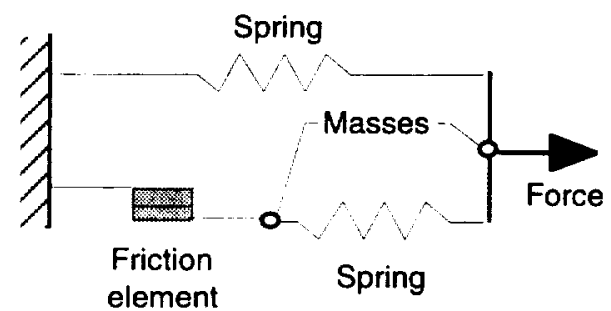

Fig. 12 Simplified dynamic model of joint.

Currently, it is believed that the initial net microlurch response of seven microns results from either the latch joint, the revolute joints, or all joints releasing internal strain energy to obtain a lower total energy state than is present at the end of deployment. The random micro-lurches after the fifth impulse define an "equilibrium zone" within which the test article tends to remain quasi-stable over time. The width of this equilibrium zone is about three microns, and is a measure of the kinematic accuracy of the metering truss test article. No other mechanically deployable structure found in the literature has been demonstrated to be this kinematically accurate. This accuracy is close to the absolute precision requirement for science instruments operating in the teraHertz range, making the deployment of such instruments feasible at this preliminary stage of development.

\section{Concluding Remarks}

To date, dimensional uncertainties associated with nonlinearities in deployment mechanisms have limited the accuracy of deployable reflectors to approximately
100 microns. Although this accuracy is acceptable for low-frequency $(\leq 20 \mathrm{GHz})$ communication antennas, it is unacceptable for high-frequency $(\geq 100 \mathrm{GHz}$ ) science instruments. Achieving substantial improvement in deployment accuracy requires significant advancements to be made in the load-cycle linearity of precision deployment mechanisms, and the understanding of submicron-level load-cycle nonlinearities of these mechanisms.

The revolute joint described in the present paper is designed to minimize all forms of load-cycle nonlinearity, especially freeplay, which cannot be compensated for through active control. Componentlevel test results presented herein prove that the concept is sound; exhibits no freeplay, bilinearity, or changing stiffness; and has only about two percent hysteresis in the load-cycle response. These results prove the joint to be substantially more linear, as presently designed, than any joint found in the literature to date.

A deployable reflector metering truss incorporating four of these high-precision revolute joints was fabricated and tested. These tests identify a new nonlinear response phenomenon referred to herein as "micro-lurching" which is a micron-level change in the equilibrium shape of a structure following a transient dynamic disturbance. At present, it appears that microlurching is caused by load transmission through contact friction within the revolute joints, the end-ofdeployment latch joint, or both. However, additional analyses and tests are necessary to characterize these relationships fully as well as the effect of gravity on micro-lurching. These investigations are very important because the stochastic nature of microlurching might ultimately prove it to be the response parameter that defines the kinematic accuracy of a precision mechanical-deployable structure. Although friction-induced hysteresis and micro-lurching are probably unavoidable in deployable structures because of the complex load paths within the mechanical joints, it appears to be possible to reduce these undesirable effects to an acceptable level by minimizing load transfer through friction.

The present results indicate that it is both possible and practical to design deployable structures that have a kinematic accuracy of a few microns. No other mechanically deployable structure found in the literature has been demonstrated to be this kinematically accurate. This accuracy is close to the absolute precision requirement for science instruments operating in the teraHertz range, making the deployment of such instruments feasible at this preliminary stage of development. 


\section{References}

'Peacock, K., and Long, K. S., "Astronomical Telescopes: A New Generation," Johns Hopkins APL Technical Digest, Vol. 10, No. 1, 1989.

${ }^{2}$ Miller, R. K., Thomson, M. W., and Hedgepeth, J. M., "Concepts and Analysis for Precision Segmented Reflector and Feed Support Structures." NASA CR 182064, December 1990.

${ }^{3}$ Rhodes, M. D., "Design Considerations for Joints in Deployable Space Truss Structures," presented at the First NASA/DOD CSI Technology Conference, Norfolk, Virginia, November 18-21, 1986.

${ }^{4}$ Belvin, W. K., "Modeling of Joints for the Dynamic Analysis of Truss Structures," Master's Thesis submitted to The School of Engineering and Applied Science of the George Washington University, December 1985.

${ }^{5}$ Chapman, J. M., Shaw, F. H., and Russell. W. C., "Dynamics of Trusses Having Nonlinear Joints," presented at the Workshop on Structural Dynamics and Control Interaction of Flexible Structures, NASA Marshall Space Flight Center, Huntsville, Alabama, Anril 22-24. 1986.
Viscoelastic Joint Actuators," Joumal of Sound and Vibration, Vol. 143, 1990, pp. 407-422.

${ }^{8}$ Salama, M., Umland, J., Bruno, R., and Garba, J., "Shape Adjustment of Precision Truss Structures: Analytical and Experimental Validation," Smart Materials and Structures, Vol. 2, 1993, pp. 240-248.

${ }^{9}$ Wada, B. K., Fanson, J. L., and Chen, G.-S., "Using Adaptive Structures to Enable Future Missions by Relaxing Ground Test Requirements," Journal of Spacecraft and Rockets, Vol. 28, No. 6, NovemberDecember 1991, pp. 663-669.

${ }^{10}$ Mindlin, R. D., "Compliance of Elastic Bodies in Contact," Journal of Applied Mechanics, Vol. 16, September 1949, pp. 259-268.

${ }^{11}$ Hachkowski, M. R., "Friction Modeling of a Revolute Joint for a Precision Deployable Spacecraft Structure," Master's Thesis submitted to the University of Colorado Graduate School, August 1995.

${ }^{12}$ Brotini, M., Barbis, A., Carli, B., Fabbrizzi, F., and Spicci, V., "High Precision Double Four Bar Linkage Mechanism for Interferometric Linear Scanning," Proceedings of the Fifth European Space Mechanisms and Tribology Symposium, 1993, pp. 



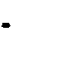

- 
. 